\title{
ENTREVISTA A JULIO PAZOS ${ }^{1}$
}

\author{
POR \\ GLADYS JARAMILLO \\ Universidad Central del Ecuador, Quito
}

Cuando la Casa de las Américas otorgó a Julio Pazos, en 1982, el primer premio de poesía por su libro Levantamiento del país con textos libres, su obra lírica ya gozaba del reconocimiento de la crítica ecuatoriana, pero sólo a partir de entonces cobró la dimensión internacional que, a mi entender, se merecía. Nacido en Baños (provincia de Tungurahua) en 1944, Julio Pazos publicó su primer cuaderno de poesía, Plegaria azul, cuando tenía diecinueve años. La producción posterior a ese «modesto cuadernillo» (palabras del autor) incluye Ocupaciones del buscador (1971), Prendas tan queridas las palabras entregadas al vuelo (1974), Entre las sombras las iluminaciones (1977), La ciudad de las visiones (1980; Premio Nacional de Literatura Aurelio Espinosa Pólit 1979). Al galardonado Levantamiento le sucedieron Oficios (1984), Contienda entre la vida y la muerte (1985) y un poemario todavía inédito que lleva el título provisional de «Mujeres».

Además de poeta, Julio Pazos es un estudioso de la literatura, asignatura que enseña en la Pontificia Universidad Católica del Ecuador. Interesada por conocer su manera de ver su propia poesía, en abril de 1987 le hice una entrevista, de la cual doy ahora a conocer algunos segmentos relativos a temas tales como la función de lo autóctono en su obra, su lugar en la lírica ecuatoriana e hispanoamericana, la responsabilidad social

${ }^{1}$ La entrevista tuvo lugar en Quito en abril de 1987. No toqué en ella ciertos temas de evidente interés porque Pazos había expresado ya su opinión sobre ellos en otras entrevistas que tuvieron amplia circulación en el Ecuador. Sin embargo, para beneficio de los lectores de otras latitudes, añadiré al presente texto, en forma de notas, referencias a algunas de dichas entrevistas. Asimismo, y con igual propósito y manera, aludiré oportunamente a la opinión de otros críticos. Los datos completos de esas fuentes puedert encontrarse en la bibliografía anexa sobre Julio Pazos. 
del poeta y otros asuntos de importancia semejante. Creo que los conceptos vertidos por Julio Pazos en esa ocasión iluminan zonas fundamentales de su obra.

Gladys Jaramillo: ¿Qué sitio le das a «la realidad» cuando escribes? ¿Tiene tu poesía un marcado referente real?

Julio Pazos: Creo que mis textos tienen un marcado referente real, pero entendiéndolo de este modo: aglutinan muchas informaciones de la experiencia cotidiana; por ejemplo, en un mismo texto pueden aparecer estas informaciones:

1) Colores tomados de las flores, las plumas y las prendas de vestir. 2) Una acción de un personaje. 3) Una reflexión sobre el sentimiento de la contingencia. 4) Indicios de problemática social. 5) Un tratamiento de los pronombres para organizar un diálogo. 6) Cierta sensorialidad auditiva, implícita o explícita. Etc.

Las otras informaciones se me escapan: traumas, neurosis, euforias, etcétera. De manera que el conjunto, a primera vista, provoca ambigüedad. Algunos lectores seleccionan las informaciones que fueron controladas por mí; otros descubren informaciones que no fueron controladas; otros relacionan las informaciones desde diferente perspectiva. Pero siempre, por cualquier código, se llega a la referencia real. Si la aglutinación de códigos produce el efecto de lo que ilaman imaginación, creo que ella, en un momento dado, nos devuelve a la realidad.

Es muy importante el referente real; me parece que en la vinculación del autor y la realidad se encuentran los elementos que nutren la poesía. $\mathrm{Si}$ entendemos por realidad las numerosas relaciones del hombre y la sociedad, del hombre y los objetos, del hombre y la interpretación que dé a los fenómenos, estas relaciones especialmente tratadas son el texto artístico ${ }^{2}$.

G. J.: ¿Qué piensas sobre el lector? ¿Quiénes son tus lectores? ¿Quién es tu lector ideal?

${ }^{2}$ Conviene tener presente la siguiente puntualización hecha por el poeta en una entrevista realizada por Francisco Febres Cordero: "Ya desde La ciudad de las visiones manifesté mi preocupación por ver la realidad ecuatoriana con todo lo que ella tiene de más duradero, con lo que viene del pasado y se proyecta hacia el presente y el futuro: esos modos de ser, esas prácticas, esas costumbres, esas presencias lingüísticas. $\mathrm{Y}$ a esos elementos los he visto con eurofia y no con tristeza, aunque parezca contradictorio... pero cuando contemplo a alguien haciendo un tapial, golpeando la tierra con el pizón, tras la miseria veo a un hombre que ejerce, con dignidad, un trabajo. Y lo respeto. Y lo canto porque ahí está el valor del ser humano. He tratado de quitarme los velos que me ha puesto la educación organizada y ver más allá de la realidad que tengo enfrente.» 
J. P.: Nunca he pretendido alcanzar la atención de numerosa cantidad de lectores. Objetivamente, los lectores de poesía siempre fueron pocos.

Tengo lectores muy próximos y que están altamente motivados por la poesía; también he encontrado lectores en los estudiantes universitarios y en los de secundaria. Entonces mi posible lector proviene de la pequeña burguesía. Este lector tiene experiencia directa o indirecta de lo agrario, tiene gran cohesión familiar, fue sometido a una educación anacrónica (quizás el hábito de la lectura sólo se encuentra en individuos de esta clase).

A veces, por la ambigüedad de mis textos, pienso que pueden liegar a lectores de otras clases y de otras latitudes.

Mi lector ideal sería un individuo curioso, sensible a la problemática del hombre, que no oculte sus sentimientos y que reciba los mensajes de la sensorialidad; un lector sin prejuicios frente a la lírica.

G. J.: ¿Cuál es tu concepto de poesía?

J. P.: Cuando tenía veinticinco años mis apreciaciones del poema y de la poesía eran inefables. Poco a poco fui distanciando el fenómeno; durante estos veinte años, gran parte del tiempo lo he dedicado al estudio del arte verbal, específicamente de la lírica. Nunca he buscado definiciones habituales; mi interés se ha concentrado en los procedimientos, en la composición, en los efectos y en tantas otras exigencias.

De igual modo, de una caótica aproximación a la realidad, he ido, lentamente, hacia un conocimiento más organizado - sólo eso-, no puedo decir profundo y menos apodíctico. Un conocimiento en donde la intuición resplandece como un bello pájaro semiciego.

G. J.: Samuel Guerra ha dicho acerca de Levantamiento que es un libro que nos hace vibrar con la "esencia poética de lo cotidiano» ${ }^{3}$. ¿Cuál es, para ti, la problemática fundamental de ese poemario?

${ }^{3}$ Esta es la acertada valoración que Samuel Guerra ha hecho de Levantamiento del país con textos libres: «Pazos ubica allí (en la formulación poética de la realidad) su tarea para hacernos vibrar con la esencia poética de lo cotidiano. En primer lugar, el poeta intuye o capta y define (con los recursos de la poesía) el condicionamiento que la realidad histórica impone para llegar a sus núcleos poéticos auténticos, a los cuales no se puede acceder si no se da como condición previa una vuelta hacia lo propio, una re-cuperación de nuestra realidad, un re-encuentro con nosotros mismos, un re-conocimiento de nuestros modos de ser, una re-valorización de nuestras costumbres. El prefijo latino 're' se convierte curiosamente en una sílaba clave que significa (tanto en poesía, como en filosofía, como en historia, como en antropologia) 'volver a'. El volver a lo nuestro es, en este momento de nuestros procesos históricos, la llave de la autenticidad» (Los designios poéticos de Pazos, p. 77). 
J. P.: En una palabra, la identidad. Desde el siglo xvirI, la preocupación por el modo de ser americano ha interesado a muchos artistas; este interés se ha articulado con la ideología, la política, la historia, los signos... En los diversos momentos, la actitud frente al problema se ha expresado con distintos matices; en nuestros días se define como la voz profunda de la comunidad americana, que ciertamente es una voz muy compleja. El artista de hoy habla con esa voz; habla desde el interior de su pueblo. Habla, específicamente, desde el pueblo más pobre, porque ha encontrado que en él se entrecruzan los más remotos antecedentes. Creo que el texto que he generado tiene mucho de este elemento; mi intención fue ésta, pero las realizaciones se limitan a la capacidad que tengo ${ }^{4}$.

G. J.: ¿Cómo ubicas tu poesía en el panorama nacional?

I. P.: Entiendo que los postmodernistas (Jorge Carrera Andrade, Gon-

${ }^{4}$ Uno de los temas principales de Levantamiento es el rescate lírico de las comidas tradicionales del Ecuador, como ilustra muy bien el poema reproducido a continuación (con el debido permiso del autor y de la Editorial El Conejo), que tiene que ver con la «colada morada», infaltable en las mesas ecuatorianas en el Día de Difuntos.

ESPIRITU DEL MAIZ AMATISTA

Pones ishpingo.

Dejas dos días la harina negra humedecida en el

Está a punto cuando un pájaro amatista tiemble pongo. en su interior.

Para que no pierda el espiritu pones algún resplandor de los ojos de tu madre

$y$ todo el aire que puedas conseguir en las

Para que gane contemplación, cordilleras.

para que sea como una piedra que canta pondrás tus levitaciones.

Después, ya no te preocupes; vendrán en la sombra las abuelas con sus mantillas perfumadas y dejarán en la amatista del maíz sus quedos pies y los caminos atrapados de los abuelos, también desintegrados y fulgurantes.

(De Levantanienio...)

Valga aquí señalar que Julio Pazos no sólo escribe sobre nuestras comidas, sino que además las cocina excelentemente (sus famosas recetas son a menudo publicadas en prestigiosas revistas). En su casa, definitivamente, las pailas no están de adorno. 
zalo Escudero, Miguel Angel León, etc.) fueron de la observación de la realidad próxima hacia un cosmopolitismo (Carrera), o hacia un adensamiento de la individualidad (Escudero), o hacia una problemática marcadamente social (Zambrano). En cambio, ciertos autores que vienen después de 1950 (Jorge Enrique Adoum, Antonio Preciado, Fernando Nieto, Javier Ponce, Iván Carvajal) parten de la realidad próxima y van hacia el descubrimiento de lo mítico de esa realidad, hacia las fuerzas ancestrales, hacia el encuentro del paisaje en la psicología del habitante. Me parece que, por mis intenciones, me ubico en esta problemática.

G. J.: ¿Y dentro del contexto poético del continente?

J. P.: En líneas muy generales podríamos señalar tres corrientes por las que está desarrollándose actualmente la lírica hispanoamericana: 1) La «antipoesía», propuesta - entre otros- por Nicanor Parra. 2) La «poesía conversacional», cuyo más alto exponente es Ernesto Cardenal. 3) El «nuevo realismo», como denomina Roberto Fernández Retamar a una suerte de síntesis que resulta de las dos anteriores.

Yo localizaría mis textos en esta tercera corriente, aclarando que comparten especialmente las características de la línea conversacional.

G. J.: ¿Cómo ves la relación entre forma y contenido en tu poesía?

J. P.: Se dice que la poesía es, esencialmente, forma. Pienso que sí, que uno adquiere el oficio con la práctica y con la observación. Yo escribo durante dos o tres horas diarias y he logrado tener cierta "facilidad" para combinar palabras y darme cuenta de los paradigmas y los sintagmas, por el tiempo que he dedicado a ello. Pero todo eso quedaría en el aire si no viniera en ello una voluntad absoluta de ser pueblo. Eso está detrás de la creación. Me siento ligado a la mayoría, al albañil, al cargador, al tapialero, al jornalero, al fulano que se embarca en un bus, se va a Baeza y muere; al conscripto, al comerciante de baratijas, al dueño de una pequeña cantina. No tengo nada que ver con la cúspide de una pirámide ${ }^{5}$.

G. J.: Aparte de este tema vasto y envolvente de testimoniar tu época, tu realidad, de solidarizarte con los marginados, con la base de la pirá-

${ }^{5}$ Esclarecedora es a este respecto la siguiente aseveración hecha por Pazos a Francisco Febres Cordero: «Cuando comencé a escribir tenía ideas respecto al valor autónomo de la palabra, y tendía a gozarme yo solo con lo que escribía, a sentir placer con ciertos efectos que lograba crear; pero luego comprendí que eso era algo insustancial y que la poesía no es - no puede ser- algo netamente decorativo. Entonces me fui ubicando frente a la verdadera responsabilidad como creador, como poeta; como ser inmerso en una realidad concreta, que es la que me ha tocado vivir. El poeta no es ni un iluminado ni un neurótico, sino alguien que puede ver y sentir los problemas de su sociedad, que padece los conflictos de la clase a la que pertenece y sufre las presiones de otras clases, que aspira a un mundo mejor, más justo y más equitativo.» 
mide, ¿qué otros temas y «demonios» te han obsesionado? ¿Cuáles se encuentran presentes en tu producción poética?

J. P.: Sólo puedo hacer una lista general de temas: La oposición vidamuerte. La euforia que provocan los sentidos. Las diferentes violencias: guerras, explotación, tortura. La idea de un mundo mejor (paraíso). El consuelo de la imaginación. El cuerpo de la mujer; el sexo. El significado de los alimentos. El trabajo, los oficios, las artesanías. El arte: música popular y autóctona; la plástica. El tiempo. Las anormalidades físicas y psicológicas. La vida hogareña. La familia y los familiares. El amor.

«Demonios»: La revelación del lugar natal. La ciudad de Quito. La experiencia de Francisco de Asís. El dolor. La destrucción de los objetos. El conflicto burgués.

G. J.: Julio, hasta aqui hemos conversado $-y$, como en toda conversación, un poco asistemáticamente, y a la vez de forma viva- de qué escribes, por qué y para quién lo haces. ¿Qué te parece hablar ahora del cómo de tu poesia? ¿Cuáles crees que son tus constantes expresivas? ¿Cuáles los cambios en el uso de los elementos formales?

J. P.: Intenté, en mi primera juventud, producir versos clásicos. Luego. pasé a los «versos blancos», y por fin di en las lineas poéticas. Más resueltamente las líneas poéticas aparecen en La ciudad de las visiones. Entiendo que estos cambios respondieron a necesidades expresivas y a una mayor actualización en el arte verbal.

Si el ritmo se tornó prosístico (como en el diálogo o en la conversación), en cambio me entregué con énfasis a la imagen. Pero otros modos. me enriquecieron: diálogos imaginados, descripción de personajes, relatos. esbozados, espacios que entrecruzan la referencia real y la imaginación. No obstante, pienso que algunos de mis textos son discursivos y grandilocuentes. En ocasiones he regresado a la metáfora insólita. He trabajado también con los indicios - por la lectura de Antonio Machado- para provocar acumulaciones emocionales. También he dejado los textos abiertos, tratando de sugerir.

G. I.: ¿Y la Academia de la Lengua?

J. P.: La Academia tiene un papel importante en la utilización de la lengua natural, mas nada tiene que hacer con textos artísticos. En este sentido, el vocabulario, la sintaxis, el ritmo no funcionan de acuerdo a las. exigencias de la lengua natural: funcionan de acuerdo a las exigencias de la comunicación artística. Por ejemplo, el empleo de «vocablos autóctonos», que en la lengua natural significaría un desconocimiento de vocablos. hispánicos para tales o cuales necesidades del usuario, en el texto artístico significa una valoración de la cultura popular o una reacción contra los 
ideales de la burguesía. Como se ve, el mismo vocablo tiene distinto significado de acuerdo al sistema en el que se lo utilice ${ }^{6}$.

G. J.: ¿Qué opinas de la crítica? ¿Cómo recibes la crítica de tus obras?

J. P.: Me preocupa la crítica del lector próximo; ellos descubren imperfecciones y aciertos; ellos subrayan los ripios, los hermetismos, las confusiones, etc.

La crítica sistemática me sirve para realizar lecturas de mis propios textos, pero no me ha servido para reorientar mi trabajo.

Quisiera terminar estas páginas reproduciendo unos versos de La cilldad de las visiones, que revelan elocuentemente la dimensión humana y rebelde del poeta Julio Pazos:

cómo me gustaría escribir todo al revés

los clásicos al revés

los caballos surrealistas al revés

cómo me gustaría desvivir

hacerlo de nuevo

desvivir el tiempo para colocar el fuego fuera de la cocina

caminar el tiempo al revés para asustar a la gente

cómo me gustaría caminar por los tumbados

y hablar con las autoridades al revés

y solo mirar el mar al derecho

cómo me gustaría ser feliz al revés

que mis hijos sean mis padres

que mi mujer sea yo

${ }^{6}$ Simón Espinosa, en iluminador estudio sobre Levantamiento, se refiere a lo que Pazos llama «vocablos autónomos». Anota previamente que en el libro hay «una evidente intención social no fabricada con denuncias, ni con descripciones surrealistas, ni con un lenguaje dictado por los dogmas. Es el lenguaje de las cosas conocidas por el pueblo, de los gestos amados por la comunidad, es el sabor de las raíces (...)». Añade luego que por ello, porque Pazos ha logrado ecuatorianizar la literatura, «las pailas, comidas y sabores tienen insospechada trascendencia histórica en la poética ecuatoriana (...). Hay elaboración de un lenguaje poético propio, adaptado a este tiempo, creado para esta circunstancia histórica nuestra, enraizado en lo social, con evidente intención política, sin que por esto último haya sufrido mengua alguna la independencia del lenguaje como materia de lo poético (...). El libro de Pazos, nacido del amor a lo que nos identifica (...), ha creado un lenguaje con significado (...), nos ha permitido nombrarnos, identificarnos, aceptarnos. En este sentido, este pequeño libro de poemas tiene una gran importancia sicológica, social y filo. sófica» (Pazos, Un lenguaje poético propio, III). 
cómo me gustaría poner las comas

en los lugares no previstos por la gramática

y morder a los perros

y perseguir a los dictadores

recostarme al revés

finalmente morirme al revés

(De La ciudad de las visiones.)

\section{BIBLIOGRAFIA SOBRE JULIO PAZOS}

Ansaldo, Cecilia: Programa «Diálogo con un escritor». Invitado, Julio Pazos Barrera, Guayaquil, diciembre 1986.

ArIas M., Rafael: «Julio Pazos Barrera: el poeta que regresa», El Tiempo (Quito), 5 de febrero de 1978, p. 2.

ArIas, Raúl: «Conversación con Julio Pazos Barrera», La Revista de Hoy (Quito), 13 de marzo de 1983, pp. 5-7.

Artieda, Fernando: «Julio Pazos, poeta del Ecuador profundo», Ariel (Guayaquil), 40 (marzo 15, 1982), 77.

Bermeo, María, et al.: Visión critica de la poesía de Julio Pazos Barrera, Tesis de Licenciatura, Ambato, 1986.

Cordero de Espinosa, Susana: «Los motivos de lo cotidiano en Levantamiento del país con textos libres y oficios, de Julio Pazos B.», ponencia, III Encuentro sobre Literatura Ecuatoriana, Cuenca, noviembre 1984.

Cordero de Espinosa, Susana: «Lectura de fin de año», Hoy (Quito), diciembre de 1985, p. 4-A.

Dávila Vázouez, Jorge: «La poesía de Pazos: un mundo sensorial», El Comercio (Quito), 5 de abril de 1983, p. 5-A.

DÁvila VÁzquez, Jorge: «Diez poetas», Hoy (Quito), 20 de abril de 1984, p. 4-A.

DÁvila VÁzQuez, Jorge: "Las ocupaciones más humildes», Hoy (Quito), 20 de septiembre de 1984, p. 4-A.

Escudero, María: «La poesía, a propósito de Levantamiento del país...», El Comercio (Quito), 20 de febrero de 1983, p. 1-B.

Espinosa, Simón: «Pazos: un lenguaje propio» (I), Suplemento Cultural de El Comercio (Quito), 22 de mayo de 1983, p. 5.

Espinosa, Simón: «Pazos: un lenguaje propio» (II), Suplemento Cultural de El Comercio, 29 de mayo de 1983, p. 8.

Espinosa, Simón: «Pazos: un lenguaje poético propio» (III), Suplemeno Cultural de El Comercio (Quito), 5 de junio de 1983, p. 8.

Espinosa, Simón: «El árbol del paraíso», Hoy (Quito), diciembre de 1985, p. 4-A (del archivo personal del autor).

Febres Cordero, Francisco: «Julio Pazos», Hoy (Quito), 7 de agosto de 1982, p. 7-A.

GarcíA, Mariana Cristina: «El poeta Julio Pazos Barrera», Suplemento dominical de El Comercio (Quito), 1980, p. 10.

García, Mariana Cristina: «Oficios», El Comercio (Quito), 7 de abril de 1985, p. 1-B. 
GonzÁlez, Otto-Raúl: «Premio Casa de las Américas: Poesía de Julio Pazos», Excelsior (México), 26 de abril de 1983, p. 6.

GonzÁlez Poyatos, José: «Entre las sombras las iluminaciones», Mensajero (Quito), 627 (enero 1978), 11-13.

Guerra B., Samuel: «Consideraciones marginales», Hoy (Quito), 18 de enero de 1984, p. 4-A.

Guerra B., Samuel: «Los designios poéticos de Pazos», El guacamayo y la serpiente (Cuenca), 26 de octubre de 1986, pp. 72-85.

Hidalgo de Velasco, Laura: «Una visión de La ciudad de las visiones», Mensajero (Quito), 652 (junio 1980), 9-11.

Jaramillo, Gladys: "Los Oficios de Julio Pazos», prólogo, en Julio Pazos, Oficios (Quito: Casa de la Cultura Ecuatoriana, 1984), pp. 5-16.

Jaramillo, Gladys: «Oficios», Diners (Quito), 26 de mayo de 1984, p. 75.

JarAmillo, Gladys: Entrevista a Julio Pazos, Quito, abril 1987.

Papastamatíu, Basilia: "Mutaciones del ser y de la imagen», Casa de las Américas. (La Habana), 136 (1983), 167-170.

Parral de TerÁn, Esthela: «Nueva poesía ecuatoriana», El Tiempo (Quito), 25 de junio de 1978 , p. 3.

Parral de Terán, Esthela: «La ciudad de las visiones», Suplemento dominical de El Comercio (Quito), 20 de julio de 1980, p. 10.

Rodríguez CASTelo, Hernán: Lírica ecuatoriana contemporánea, tomo 2 (Bogotá: Círculo de Lectores, 1979), pp. 624-626.

Rodríguez Castelo, Hernán: Literatura ecuatoriana 1830-1980 (Otavalo: Instituto Otavaleño de Antropología, 1980), p. 141.

Rodríguez Castelo, Hernán: «El 'Levantamiento' y su clave», Hoy (Quito), enero de 1983, p. 4-A.

Rodríguez CASTElo, Hernán: «1986: buen año para la lírica», Expreso (Guayaquil), marzo de 1987 (del archivo personal del autor).

Rodríguez CAstelo, Hernán: «Lírica del 86: la Contienda», Expreso (Guayaquil), marzo de 1987 (del archivo personal del autor).

Rota, Gabriela, y Margarita Graetzer: Entrevista a Julio Pazos, 1986 (del archivo personal de Julio Pazos).

Salvador Lara, Jorge: "Un poeta premiado en Cuba», El Comercio (Quito), 23 de junio de 1983, p. 5-A.

Torres, Pancho: «La poesía actual en el Ecuador», Uso de la palabra (Babahoyo), sin fecha, p. 36.

«Crítico ecuatoriano comenta obra de poeta ecuatoriano, en España», El Tiempo (Quito), 12 de octubre de 1971, p. 6.

«Con Julio Pazos, Premio Aurelio Espinosa Pólit», El Tiempo (Quito), 26 de noviembre de 1979 , p. 9.

«Versos musicales de Julio Pazos en el TEUC», El Tiempo (Quito), 19 de agosto de 1982, p. 11-A.

«Oficios», por Julio Pazos Barrera. Hoy (Quito), 10 de julio de 1984, p. 6-B. 
